\title{
Anesthesiologists Role in Management of Major Arterial Injuries during Endonasal Endoscopic Skull Base Surgeries
}

\section{Eroglu T.N. ${ }^{*}$, Meco B.C. ${ }^{*}$, Kahilogullari G. ${ }^{* *}$, Bozkurt M. ${ }^{* *}$, Beton S. ${ }^{* *}$, Yorukoglu D. \\ *Ankara University School of Medicine, Dept of Anaesthesiology \& Intensive Care, Ankara, Turkey \\ ${ }^{* *}$ Ankara University School of Medicine, Neurosurgery, Ankara, Turkey \\ ${ }^{* * *}$ Ankara University School of Medicine, Otolaryngology, Ankara, Turkey}

Recently endonasal endoscopic surgery (EES) became a standard approach for pathologies along the skull base (1). Nevertheless, regardless of the approach operating in this area could risk neurovascular injuries (1). Due to close anatomical relationship in sphenoid sinuses, internal carotid artery (ICA) injury may happen at any stage of surgery. This case report focuses on the management of our only ICA injury in our endoscopic skull base unit ( $>700$ cases).

Case report: In a 44 year old female EES was planned for pituitary adenoma resection. Routine ASA monitorisation and anesthesia induction was performed. And, an invasive radial artery monitorisation, and a $16 \mathrm{G}$ iv line was placed. Intraoperatively a massive bleeding from left ICA has occurred leading hypotension. Immediately while starting norepinephrine, a femoral vein catheter was inserted, which led rapid perfusion for volume resuscitation ( $2 \mathrm{URBC}$ and $14 \mathrm{~L}$ crystalloid). As bleeding could not be controlled despite all packing efforts, an emergency surgical clipping of proximal ICA in the neck has been performed. The patient was then transferred to the angiography unit for endovascular management also showing contralateral retrograde perfusion. Nevertheless in order not to risk ophthalmic artery, the patient was taken back to operating room for transcranial clipping of distal ICA. At the end, the patient was once again transported to angiography to control leak sealing, followed by the ICU further intubated and mechanically ventilated, and was extubated in day 2 with a mild pitosis on her left eye.

Discussion: Although the ICA injury has a low incidence during EES, the response must be immediate, effective and organized. This could only be achieved through an algorithmic, well-prepared multidisciplinary team approach. The anesthesiologist plays a crucial role here in stabilizing the patient,which would facilitate time for optimal management. The anesthesiologist should be prepared for the management of unexpected massive hemorrhage as well as a multimodal approach by neurosurgeons, otolaryngologists and interventional radiologists. As such, our case needed not only transfers to the radiology unit, but also craniotomy and neck surgery for vascular sacrifice to completely control bleeding.

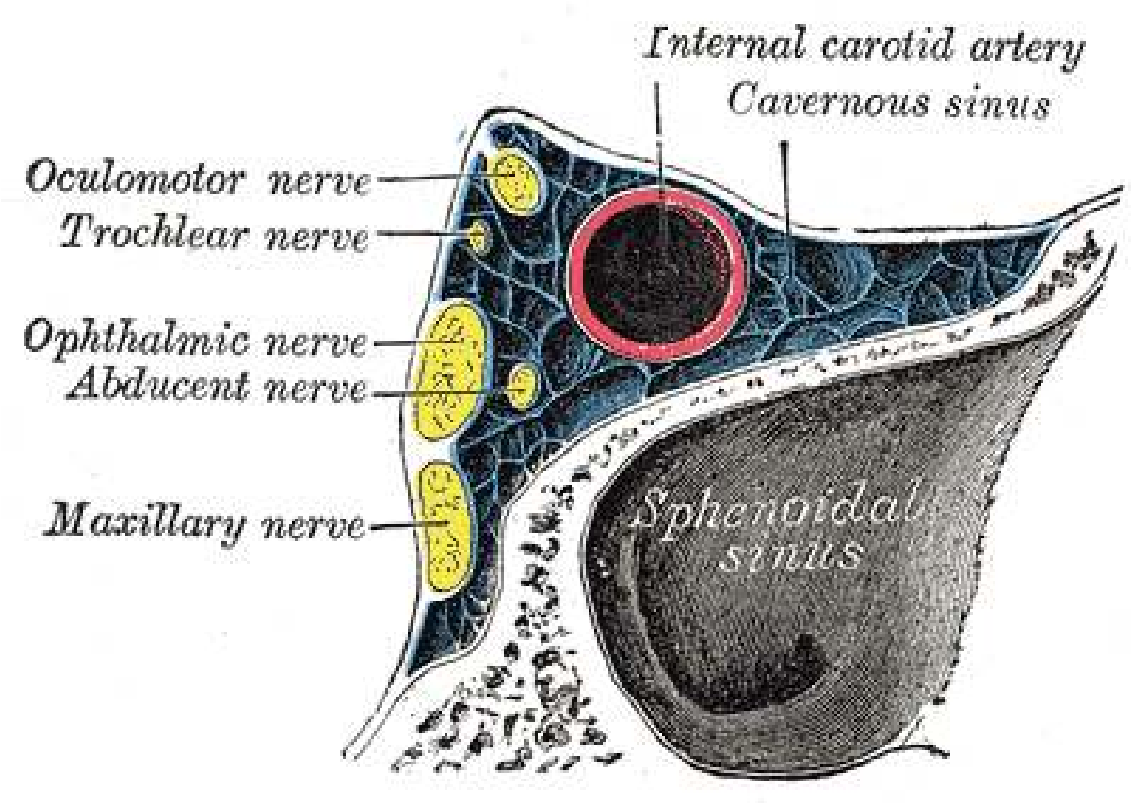

Figure 1. ICA and sphenoid sinus close anatomical relationship

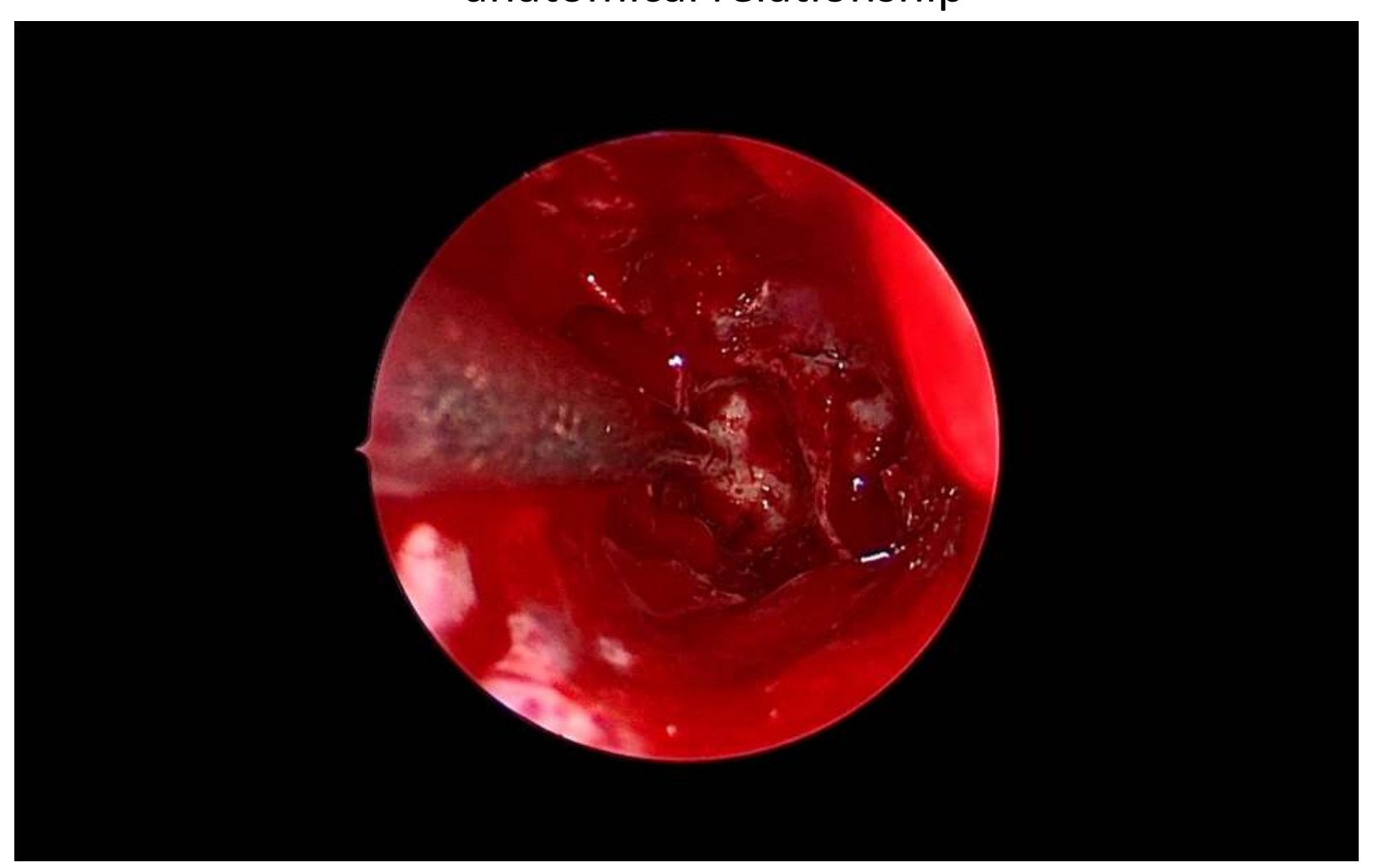

Figure 2. Endoscopic image from the surgery pre blow-out

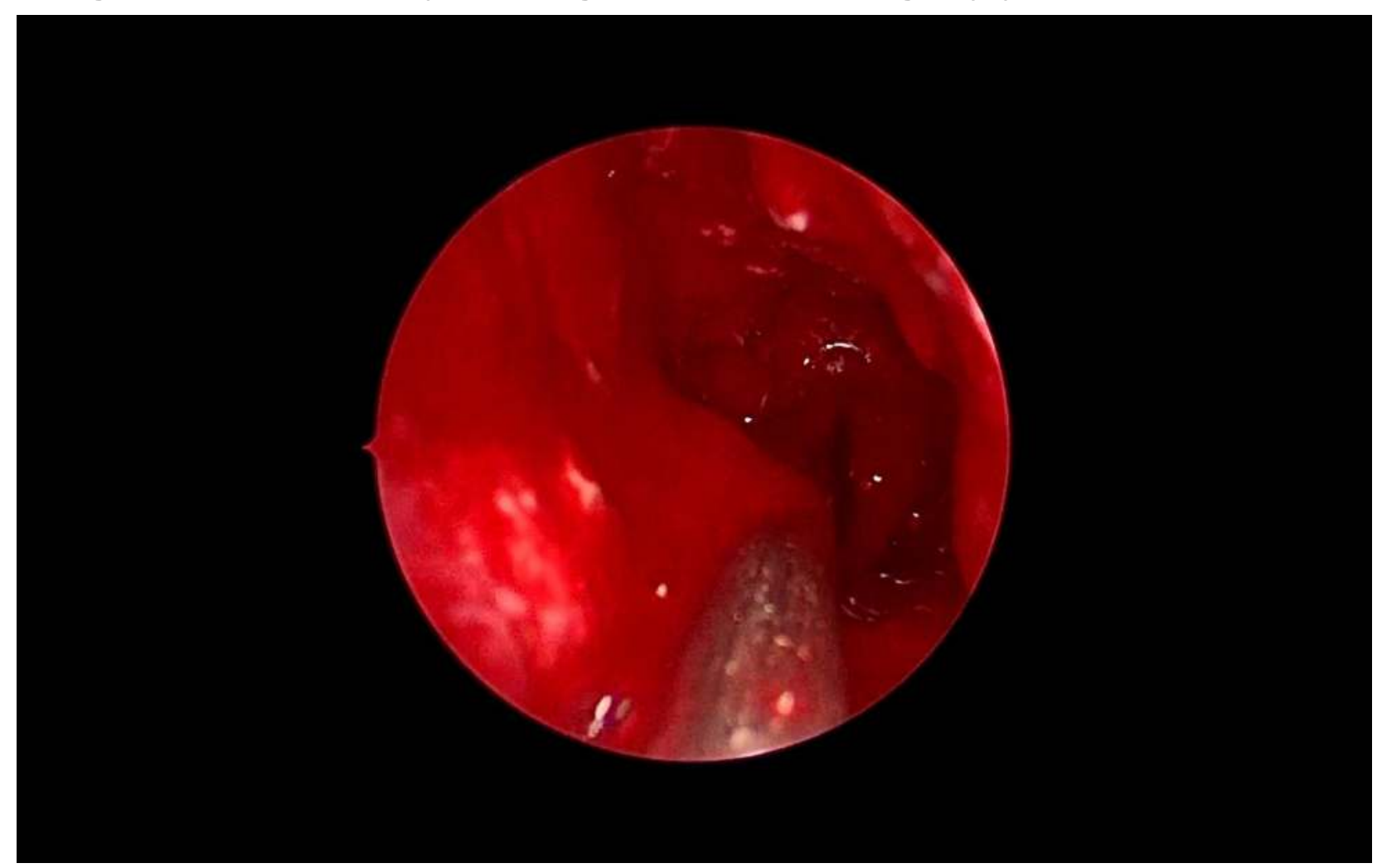

Figure 3. Endoscopic image from the surgery blow-out moment

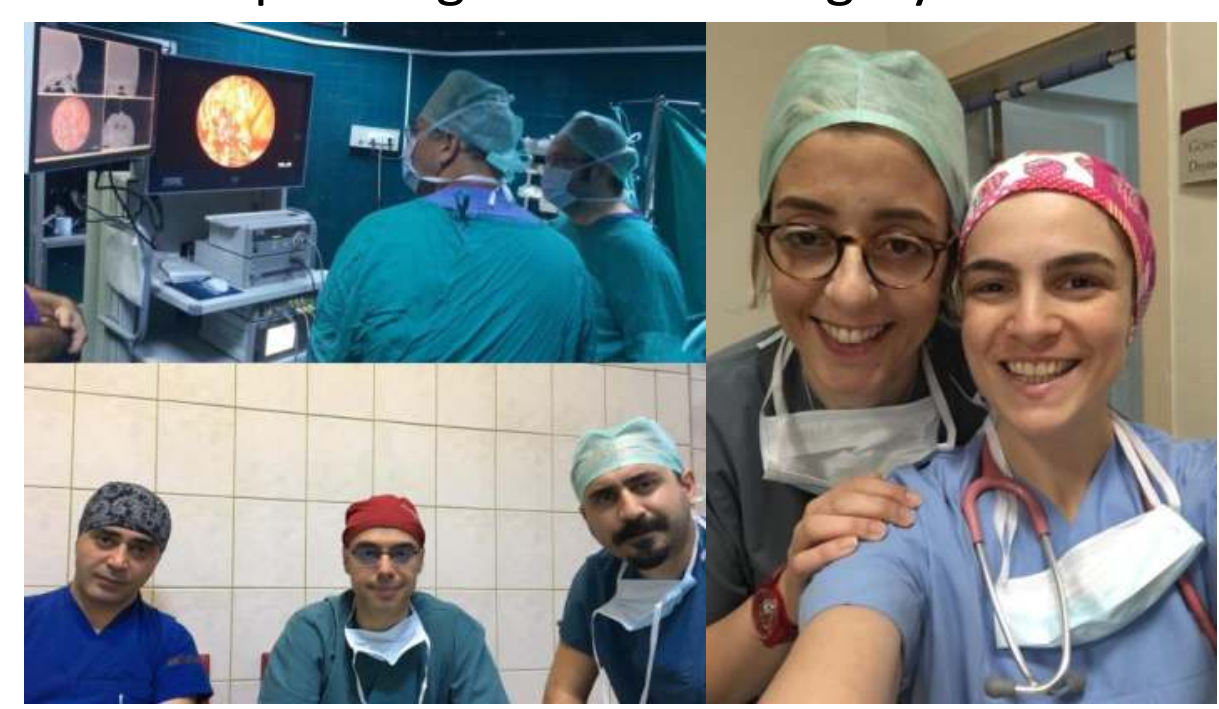

Figure 4. EES surgeon and anesthesiologist team

Learning Point: As a member of the EES team, anesthesiologists should be prepared to handle unexpected severe arterial hemorrhage especially from ICA to enable time for optimal management. 